\title{
ELEMENTOS DE RETOMA EN LA CLÁUSULA RELATIVA EN ESPAÑOL
}

\author{
THE RESUMPTIVE ELEMENTS IN SPANISH RELATIVE CLAUSE
}

\author{
ALIANA LOPES CÂMARA \\ Universidade Estadual Paulista, São José do Rio Preto \\ aliana@ifsp.edu.br
}

\section{RESUMEN}

Esta investigación estudia los elementos de retoma en la oración relativa que se presenta en la lengua española. Estudios previos apuntan a la influencia de diversos factores para el uso del elemento de retoma en la cláusula relativa (relativa no restrictiva, núcleo indefinido, atribución de función pragmática, relativización de la posición sintáctica de objeto directo). Aquí se investiga la influencia de esos factores para el uso del reasuntivo, a partir de una perspectiva funcionalista que comprende el componente gramatical como constituido por los niveles pragmático, semántico, morfosintáctico y fonológico, estructurados jerárquicamente de modo descendente. Para eso se seleccionan datos de seis variedades del español, extraídos del PRESEEA. Por medio de un análisis cuantitativo y cualitativo, se comprueba que el reasuntivo puede ser una marca morfosintáctica de la codificación de la relativa no restrictiva y puede ser soporte a la atribución de función pragmática en las relativas de sujeto.

Palabras clave: Gramática Discursivo Funcional, codificación morfosintáctica, pragmática.

\section{ABSTRACT}

This research studies the resumptives in the relative clause in Spanish. Previous studies point to the influence of various factors for the use of the resumptive element in the relative clause (non-restrictive relative clause, indefinite nucleus, attribution of pragmatic function, relativization of the direct object position). Here we investigate the influence of these factors for the use of the resumptive from a functionalist perspective that understands the grammatical component as constituted by the pragmatic, semantic, morphosyntactic and phonological levels, structured hierarchically. For that, data are selected from six varieties of Spanish, extracted from the PRESEEA. By means of a quantitative and qualitative analysis, it is verified that the resumptive can be a morphosyntactic mark of the 
codification of the non-restrictive relative clause and can be support for the attribution of pragmatic function in the subject relative clause.

Keywords: Functional Discourse Grammar, morphosyntactic codification, pragmatics.

Recibido: 25/09/2018. Aceptado: 28/05/2019.

\section{INTRODUCCIÓN}

- ste estudio ${ }^{1}$ investiga un fenómeno registrado en la lengua española, no sólo Een el registro coloquial, sino también en la escritura: la presencia, en la oración relativa, de un elemento de retoma que se refiere al núcleo nominal, como el ejemplificado en (1).

1 yo creo que si que que por ahi si se podría conseguir ¿no? I mejorar por lo menos que es algo que lo tenemos al alcance de la mano <simultáneo> ¿no? (MADR_H22_026:1.115$116)^{2}$

En este caso, la cláusula relativa que lo tenemos al alcance de la mano especifica el núcleo nominal algo y crea una imagen mental del referente. Dos mecanismos cohesivos son utilizados en la retoma del núcleo: el pronombre relativo ${ }^{3}$ que y el pronombre oblicuo lo. Esa estrategia, llamada relativa de reasuntivo, de copia pronominal o de pronombre de retoma, puede ser usada, según Cerrón-Palomino (2006), en la relativización de todas las posiciones sintácticas en español, a diferencia de la mayoría de las lenguas en las que está disponible sólo para algunas posiciones sintácticas.

A pesar de ser un fenómeno comprobado en el español, esa construcción aún es sancionada por la norma del español que "tiende a considerar incorrectas las va-

${ }^{1}$ Este trabajo forma parte de una investigación postdoctoral desarrollada bajo la supervisión del profesor Roberto Gomes Camacho, a quien le agradezco por la colaboración en la interpretación de los datos y por los comentarios sobre el texto y a los profesores Willianice Maia y Rubén Álvarez Venegas por revisar el texto.

${ }^{2}$ Los fragmentos creados no tienen citación y los fragmentos reales fueron extraídos del corpus PRESEEA y están acompañados entre paréntesis del origen del informante (en el ejemplo, Madrid), de los códigos que permiten su localización (H22), del número del texto (026) y de la línea en la que se encuentra el ejemplo (l.115-116). Los códigos se refieren al perfil de los informantes:

Primera Posición: $\mathrm{H}=$ hombre o $\mathrm{M}=$ mujer

Segunda Posición: 1=generación 1 (20-34), 2=generación 2 (35-54) o 3=generación 3 (>55)

Tercera Posición: $1=$ nivel de estudios 1 (primarios), $2=$ nivel de estudios 2 (medios), $3=$ nivel de estudios 3 (superiores)

${ }^{3}$ No se discutirá aquí la despronominalización de "que", pero el lector puede leer sobre el asunto en Trujillo (1990) y Brucart (1999). 
riantes reduplicadas" (Brucart, 1999, p. 403). La norma considera como correcta sólo la estrategia estándar, sin la repetición pronominal ${ }^{4}$ :

Varios autores (Trujillo, 1990; De Mello, 1992; Silva-Corvalán, 1996; Bentivoglio, 2003; Zabalegui, 2005; Caviglia y Malcuori, 2007; Cerrón-Palomino, 2006, 2015; Borzi y Morano, 2009; Suárez-Fernández, 2010, 2013) estudiaron el fenómeno, considerando factores pragmáticos, semánticos, morfosintácticos y fonológicos, llegando a la conclusión de que el reasuntivo es un fenómeno motivado por diversos factores, como la relativa no restrictiva, la atribución de función pragmática, la indefinición del antecedente, la función sintáctica de OD, etc.

La propuesta es revisar la influencia de algunos de estos factores, investigando la oración relativa con elemento anafórico en español, a partir de la perspectiva teórica funcionalista. Así, se intenta comprender el fenómeno y sus múltiples causas en un modelo gramatical estructurado jerárquicamente, que comprende los niveles pragmático, semántico, morfosintáctico y fonológico, a partir de una perspectiva descendente, hecho que, por lo que se sabe, no fue realizado por algún investigador del pronombre de retoma en la relativa del español.

Para este propósito, el texto se organiza de la siguiente manera: en la sección 1 se discuten brevemente los presupuestos teóricos de la Gramática DiscursivoFuncional (en adelante GDF) y cómo esta teoría comprende la formulación y la codificación de la cláusula relativa; en la sección 2 se revisan algunas propuestas teóricas elaboradas para explicar el uso del pronombre de retoma; en la sección 3 se explican los datos empleados y la metodología; en la sección 4 se presentan el análisis y la interpretación de los datos y, finalmente, en la última sección se sintetizan las principales conclusiones de este estudio y se exponen algunos desarrollos futuros para la investigación.

\section{LOS PRESUPUESTOS TEÓRICOS: LA GRAMÁTICA DISCURSIVO- FUNCIONAL}

GDF se define en el ámbito funcionalista como una teoría que considera los objetivos que el hablante tiene en la mente como determinantes de la producción

\footnotetext{
${ }^{4}$ Se exceptúan las oraciones relativas en las que el pronombre relativo está en función de complemento indirecto, como en " $\mathrm{y}$ si es una persona mayo<alargamiento/>r / un hombre o una mujer al que por ejemplo le preguntas por <vacilación/>una calle? (LHAB_H11_001:1.10-11)”, ya que dichas reduplicaciones "no derivan de la especial naturaleza de la cláusula en que aparecen, sino de condiciones más generales de la sintaxis del español, que permiten tal duplicidad en cualquier tipo de construcción” (Brucart, 1999, p. 405).
} 
verbal. A pesar del enfoque funcional, esa teoría también se preocupa de la formalización de los aspectos lingüísticos, lo que la aproxima a teorías formalistas. El esbozo general de GDF es presentado en la Figura 1.

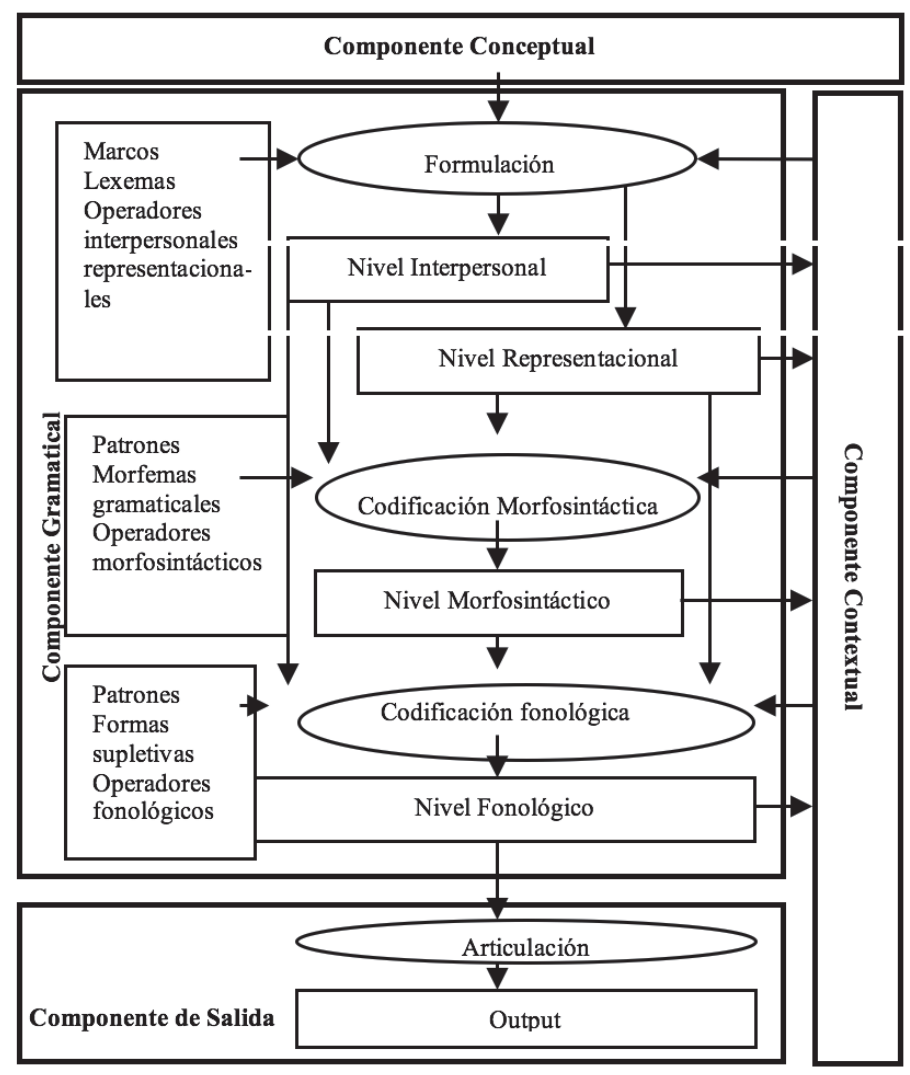

Figura 1. Esbozo general de la GD

El modelo gramatical se estructura de modo descendente, en la siguiente dirección: pragmática -> semántica -> morfosintaxis $->$ fonología. Como consecuencia, las cláusulas son elaboradas a partir de la operación de formulación, que selecciona las representaciones pragmáticas y semánticas, y de codificación, que las convierte en representaciones morfosintácticas y fonológicas.

El componente gramatical está compuesto por cuatro niveles: el interpersonal, el representacional, el morfosintáctico y el fonológico, organizados descendente y jerárquicamente.

Según Hengeveld y Mackenzie (2008), el nivel interpersonal se refiere a los aspectos formales de la unidad lingüística que reflejan su papel en la interacción 
entre el hablante y el oyente. Sus relaciones jerárquicas son el movimiento, el acto discursivo, la elocución, el hablante, el oyente y el contenido comunicado. El nivel representacional, a su vez, corresponde a los aspectos semánticos de las unidades lingüísticas e incluye los siguientes estratos: el contenido proposicional, el episodio, el estado de cosas, la propiedad configuracional y otras unidades semánticas sin relación jerárquica. El nivel morfosintáctico recibe las representaciones pragmáticas y semánticas y las convierte en una estructura morfosintáctica. Dicho nivel comprende los siguientes elementos: expresión lingüística, sintagmas y/o cláusulas. Finalmente, el nivel fonológico está compuesto por el enunciado, la frase entonacional, la frase fonológica y la palabra fonológica.

Según esta perspectiva teórica, la relativa restrictiva y la relativa no restrictiva se distinguen en los procesos de formulación y codificación. La relativa no restrictiva es formulada pragmáticamente como un acto discursivo con función retórica Aside de añadir información adicional. La relativa restrictiva, a su vez, se formula semánticamente como un modificador, cuya función es identificar un referente entre otros posibles. Para entender esa diferencia, se consideren las construcciones en (3) y (4). (SCHI_H13_073:1.55)

¿qué puedes pensar del gobierno que tú<alargamiento/> lo estás / viendo de acá de afuera? // $<[\mathrm{e}]><[\mathrm{s}]>$ tás vien $<$ palabra_cortada/ $/$ tú $<[\mathrm{e}]\rangle\langle[\mathrm{s}]>$ tás viendo una película donde $<[\mathrm{e}]>$ stás viendo $\mathrm{qu}<[\mathrm{e}]>$ están robando a ojos vistos / y no puedes hacer nada // porque como $<[\mathrm{d}]>$ icen muchos / si no hay alguien quien hable al principio siempre sigue / o / tam $<[\mathrm{b}]>i e ́ n$ está / que si le hablas al principio y si empiezas a grita $<$ alargamiento/ $>\mathrm{r} /$ a vocifera $<$ alargamiento/ $>\mathrm{r} /$ est $<[\mathrm{o}]>\mathrm{o} \quad \mathrm{l}<[\mathrm{o}]>\mathrm{otro}$ / un día te van a desaparecer (MONR_H23_066:1.10201)

La primera tiene un núcleo nominal cuyo referente no está definido de antemano en el contexto o en el conocimiento compartido por los interlocutores, por lo tanto, es necesario el uso de la relativa restrictiva que le hayan hecho algo para delimitar el referente, de manera que el oyente pueda construir una imagen adecuada de él, identificándolo entre un conjunto de referentes posibles.

En la segunda, a su vez, el núcleo nominal el gobierno retoma un referente ya conocido por los interlocutores en el momento del habla. La relativa no restrictiva que tú lo estás viendo de acá de afuera constituye un Acto Discursivo que se relaciona al otro Acto (la cláusula principal). Un argumento para defender el estatuto de Acto para la relativa no restrictiva es que ella tiene elocución independiente del Acto al que se relaciona. En (4), por lo tanto, la elocución interrogativa incide solamente sobre la información del primer acto (quépuedes pensar del gobierno). La 
información de la oración relativa tiene elocución afirmativa, lo que significa que no se cuestiona la información acerca de que se ven las acciones del gobierno como telespectador, y no como alguien que participa en las decisiones.

Otro argumento para comprobar el estatuto de acto discursivo para la relativa no restrictiva es la posibilidad de insertar elementos propios de este estrato, como el modificador "encima", que modifica el estatuto del acto dentro del movimiento en (5), o un modificador de contenido comunicado, como "supuestamente", que indica en (6) que el hablante está transmitiendo una visión de otras personas:

entonces eeh yo me encontraba con un trabajo hh // que te<alargamiento/ $>\mathrm{mmm}$ dedicaba todo el día // que <palabra_cortada/> en el que ya no aprendía absolutamente nada // que encima no me lo pagaban demasiado bien / y que no tenía perspectivas de futuro // pues les dije que dimitía / se quedar<alargamiento/> / se quedaron muy sorprendidos (MADR_H13_013:1.716)

6 A: e<alargamiento/>n / Estados Unidos yo supe que se podía viajar // y<alargamiento/ / supuestamente las pirámides se usaron para<alargamiento/ / / los iniciados en la masonería // los masones

B: $\mathrm{mjm}$

A: el sarcófago / que supuestamente $<[e]>$ stá $<[a]>$ hi<alargamiento/> / que lo robaron / el sarcófago siempre permaneció<alargamiento/> / supuestamente siempre permaneció vacío (MONR_H23_066:1.607)

Otra característica de la relativa no restrictiva, según los autores, es la posibilidad de modificar núcleos ausentes, como pronombres personales y nombres propios, lo que sólo es posible en el nivel interpersonal, ya que, si el núcleo no tiene contenido lexical, como IPA en (7) y "yo" en (8), no puede ser modificado por una oración relativa restrictiva, que es un modificador de estado de cosas del nivel representacional.

7 también estoy haciendo el <siglas: [ípa] $>\underline{\text { IPA }</ s i g l a s>~ q u e ~ e m p e c e ́ ~ e s t e ~ a n ̃ o ~}$ (MONV_H13_021:1.2)

8 pos yo que $<$ e $]>$ stuve $<[e]>n$ la / bien metido en eso / en el ambiente / sí se da (MONR_H12_016:1.183)

En el proceso de formulación de las relativas restrictiva y no restrictiva, por lo tanto, el hablante tiene objetivos distintos, que reflejan diferencias en el proceso de codificación, en el que las representaciones pragmáticas y semánticas serán codificadas en representaciones morfosintácticas y fonológicas. Según Hengeveld y Mackenzie (2008), en la codificación de la cláusula relativa no hay diferencia entre 
la restrictiva y la no restrictiva en términos morfosintácticos, ya que ambas presentan el mismo patrón estructural, en que un sintagma recluta, recursivamente, una cláusula, formando una estructura más compleja. La diferente codificación de los dos tipos de relativa, así, se implantaría exclusivamente en el nivel fonológico, en el que la relativa no restrictiva es pronunciada con contorno entonacional diferente al de la oración principal.

Este estudio ofrece una nueva visión sobre las cláusulas relativas en la medida en que identifica en qué estrato cada tipo de relativa es formulada y cómo esta distinción pragmática/semántica se refleja en el proceso de codificación. La organización jerárquica de la GDF permite comprender que la diferencia entre los dos tipos de relativa se debe a las distintas intenciones comunicativas del hablante, lo que refleja en su codificación. Ese punto de vista es innovador, al conferir a la relativa no restrictiva el papel pragmático de acto discursivo, lo que difiere de la perspectiva de la Real Academia Española (RAE) (2009), que parte de una distinción que no considera la pragmática.

Además, considerando la implicación, comprobada por diversos autores (Bentivoglio, 2003; Zabalegui, 2005; De Mello, 1992; Caviglia y Malcuori, 2007), entre el uso del reasuntivo y la relativa no restrictiva, se propone aquí que el hecho de que la relativa no restrictiva se formula como un acto discursivo con función retórica Aside en el nivel interpersonal determina el uso de la copia anafórica en el nivel morfosintáctico. Eso quiere decir que el español está codificando de manera distinta los dos tipos de relativa, no sólo en la codificación fonológica, sino también en la codificación morfosintáctica.

A continuación se resumen algunos estudios sobre el reasuntivo en la relativa en español, en cuanto a los factores que se investigan en el análisis.

\section{ALGUNOS ESTUDIOS SOBRE EL REASUNTIVO EN ESPAÑOL}

Son diversas las perspectivas a partir de las cuales la cláusula relativa fue investigada y son diversas las motivaciones presentadas para el uso del reasuntivo en esas construcciones. Cualquiera que sea el prisma a partir del cual se observa ese fenómeno lingüístico, el factor de mayor influencia para el uso del reasuntivo es la restrictividad de la cláusula relativa, y es la relativa no restrictiva el espacio privilegiado para ese uso. La cuestión subyacente que falta explicar es lo que lleva la relativa no restrictiva a propiciar el surgimiento del pronombre copia.

Del punto de vista histórico, la presencia de la copia es certificada por diversos autores, que indicaron que el reasuntivo es más frecuente en las relativas no restrictivas (Bentivoglio, 2003; Zabalegui, 2005; De Mello, 1992). Los datos de los autores confirman también que es posible que la copia aparezca en las relativas restrictivas. Según De Mello (1992), eso ocurre en contextos específicos: antecedente 
modificado por un artículo indefinido, por ningún determinante, por demostrativo o por los pronombres algunos, muchos, varios, dos o tres.

Cerrón-Palomino (2015), en su estudio variacionista del español de Lima, confirma, entre otros factores, que el reasuntivo ocurre, en general, con las relativas de objeto directo. Eso es observado no solo en los datos del autor, sino en otros datos también (Trujillo, 1990; De Mello, 1992; Silva-Corvalán, 1996; Bentivoglio, 2003). Ese uso se debe al hecho de que los pronombres de objeto directo están volviéndose marcadores de concordancia de objeto (Franco, 1993), como en los casos de objeto directo léxico antepuesto, en los que ocurre un clítico anafórico en las oraciones independientes, como en el ejemplo a continuación:

11 Entonces está organizando... las tierras de la Iglesia en la zona las ${ }_{i}$ está organizando con mano de obra de carácter feudal. (Cerrón-Palomino, 2015, p. 359)

Cerrón-Palomino (2015) afirma que es posible el uso del reasuntivo, incluso, cuando el objeto directo léxico no es un elemento dislocado a la izquierda. De esa manera, defiende que los reasuntivos de objeto directo pueden estar volviéndose marcadores de concordancia de objeto, de modo similar a la ocurrencia categórica de $l e(s)$ en las oraciones relativas de objeto indirecto.

En cuanto al uso del reasuntivo en la relativización de la posición sintáctica de sujeto, lo cual es un contexto menos favorable al uso de la copia, el autor afirma que el reasuntivo tiene la función contrastiva de "enfatizar la unicidad del antecedente que rellena la propiedad denotada por la cláusula relativa" (CerrónPalomino, 2015, p. 365), como en (12).

Me recetaron unas pastillas que esas sí son buenas.

Para el autor, ese uso del reasuntivo pone de relieve una propiedad como exclusiva del referente especificado por la relativa restrictiva, en comparación con un conjunto virtual de candidatos posibles. El autor distingue, así, dos tipos de pronombres: el pronombre reasuntivo, que sería responsable por hacer referencia al núcleo nominal, y el pronombre contrastivo, que, marcado por la partícula sí o por acento, tendría la función, en la relativa de sujeto, de establecer un contraste entre el núcleo y todos los otros referentes posibles.

De manera análoga, se defiende aquí que el reasuntivo, en las relativas de sujeto, puede recibir las funciones pragmáticas de foco, tópico o contraste (véase la próxima sección). Contribuye para esa interpretación el hecho de que, en la oración independiente del español, normalmente no hay expresión del sujeto, debido a la rica morfología verbal de la lengua. El sujeto es explícito cuando hay determinadas motivaciones, como, por ejemplo, cuando hay la necesidad de asignarle 
una lectura contrastiva (Fanjul, 2014, p. 35). Esa "lectura" enfática o contrastiva es interpretada aquí como la atribución de función pragmática.

Aún está por comprobar, por lo tanto, si el sujeto recibe función pragmática en todas las relativas de sujeto con reasuntivo, lo que demostraría que la expresión del sujeto en la cláusula relativa sería motivada de la misma manera que en la oración independiente. De todos modos, se adhiere en este trabajo a la propuesta de Cerrón-Palomino (2015), que demuestra que no es posible que se asigne la misma interpretación para el uso del reasuntivo en la relativización de las diferentes posiciones sintácticas.

Suárez-Fernández (2013) también explica las motivaciones para el uso del reasuntivo y parte de presupuestos parecidos a los adoptados por nosotros, en la medida en que su propuesta considera que los hablantes eligen determinada estructura para alcanzar objetivos específicos. De esa manera, la autora busca entender la funcionalidad del reasuntivo, determinando sus contextos de uso. Parte de la perspectiva teórica de Dassinger y Toupin (1994), según la cual las expresiones lingüísticas son condicionadas por los objetivos comunicativos del hablante y por el procesamiento lingüístico. Además, la autora se adhiere a un postulado de Gibson (1998), según el cual, el reasuntivo ocurre principalmente cuando hay varios elementos entre el antecedente y la posición que este ocupa en la cláusula relativa, facilitando el procesamiento y promoviendo economía de la memoria de trabajo.

El uso del reasuntivo, según la autora, hace que se expliciten las funciones de los elementos dependientes del verbo, equivaliendo la estructura de la relativa a la de la cláusula independiente. Pero la autora encuentra contextos de uso del reasuntivo sin una distancia relevante entre el núcleo y el pronombre de retoma, lo que lleva a la autora a investigar la calidad del material entre el antecedente y su posición en la relativa, y no solo la cantidad de palabras. Para Suárez-Fernández (2013, p. 76), las relativas con reasuntivo presentan características especiales que justifican el uso del elemento reasuntivo: sujetos explícitos, presencia de indefinidos negativos (como nadie), polaridad negativa en el verbo, elementos enfáticos de negación o afirmación, de inclusión y exclusión (incluso, ni siquiera), cuantificadores universales o de grado extremo, indicadores temporales del tipo ya, todavía, siempre, después, modalizadores enfáticos, expresión de rasgos de tipo ponderativo o valorativo sobre el estado de cosas o sobre el referente. El carácter enfático de esos elementos disminuiría la activación del antecedente, siendo necesario el uso del reasuntivo como reactivador del referente.

La autora también destaca que el uso del reasuntivo ocurre, en general, en las relativas con status semántico y pragmático especial, como las que modifican nombres en las oraciones con los verbos ser, haber y tener. Las predicaciones con el verbo ser, denominadas "copulativas de interés", tienen la forma un/una +sustantivo + relativa: 
El hablante elige esa estructura para caracterización o valorización del referente por medio de la información de la relativa, que es la única información importante, ya que es costumbre que la información del antecedente sea redundante, como una chica en el ejemplo citado.

Según Dassinger y Toupin (1994), en las construcciones presentativas con haber y tener, también se destaca la importancia de la relativa, que presenta información adicional, nueva y relevante sobre el referente, como en (14).

Mi hermana tenía una cartera blanca que siempre peleábamos por ella. (Suárez-Fernández, 2013, p. 83)

No está claro que esa relativa sea de facto restrictiva. La definición dada por la autora para esas construcciones equivale a la de la relativa no restrictiva (adición de información sobre el referente). Si el núcleo ya está especificado por el adjetivo blanca y la cláusula relativa trae un comentario adicional sobre el antecedente, ¿№ es posible clasificarla como no restrictiva? Eso por sí ya sería motivo suficiente para el uso del reasuntivo. Se volverá a discutir ese ejemplo en la sección 5 .

La perspectiva teórica de la autora se aproxima de la adoptada aquí, puesto que considera, en la organización del mensaje, aspectos pragmáticos, semánticos, sintácticos y fonológicos. Sin embargo, a diferencia de la GDF, no hay una jerarquía entre esos aspectos, lo que justifica revisar la propuesta de la autora y de los demás autores, a la luz de esa teoría funcionalista, averiguando cuál es la relación que los factores establecen entre sí y cómo están organizados en el componente gramatical. En especial, se busca comprender cuál es la influencia de los factores del nivel pragmático en la aportación del uso del reasuntivo, sea en la atribución de las funciones pragmáticas a los constituyentes de la cláusula relativa, o en la formulación de las relativas.

Como se ve en esta sección, la cláusula relativa con reasuntivo ya fue objeto de investigación de diversas perspectivas teóricas, y es la relativa no restrictiva el contexto más favorable para el uso del pronombre reasuntivo. Además, varios autores atestiguan el uso de ese pronombre en la relativa restrictiva, en especial cuando modifica a un núcleo indefinido. Algunas cuestiones son movilizadas por la discusión anterior: ¿Por qué el reasuntivo ocurre, en general, con la relativa no restrictiva? ¿Por qué ocurre con la restrictiva que modifica predominantemente núcleo indefinido? Se intenta, en este estudio, responder a esas cuestiones, a partir de la perspectiva de la GDF, teniendo en cuenta el modelo gramatical descendente y jerárquico, con la intención de comprender en qué nivel y en qué estrato se encuentra cada una de las motivaciones discutidas en la Literatura para el uso de la relativa con reasuntivo. 


\section{DATOS Y PROCEDIMIENTOS METODOLÓGICOS}

El corpus utilizado aquí fue seleccionado del "Proyecto para el estudio sociolinguístico del español de España y de América" (PRESEEA), realizado por la Comisión de Sociolingüística del X Congreso Internacional de la Asociación de Lingüística y Filología de la América Latina (ALFAL). Los materiales se encuentran disponibles en el sitio electrónico http://preseea.linguas.net/ y abarcan las comunidades de habla de los centros urbanos hablantes del español - monolingües o bilingües - con alguna heterogeneidad social. La muestra seleccionada representa las variedades habladas en España, Méjico, Chile, Cuba, Uruguay y Colombia. De cada variedad se seleccionan seis textos, lo que totaliza 36 textos analizados.

Después de la selección del corpus se empieza la lectura de los textos y la identificación de las cláusulas relativas, para analizarlas, en términos cuantitativos y cualitativos, a partir de los siguientes factores:

1. Restrictividad: a) restrictiva; b) no restrictiva. En la sección 4 se presentó a las oraciones relativas modificadas por más de un modificador, donde se argumentó que, en esos casos, la cláusula relativa puede ser analizada como no restrictiva.

2. Atribución de función pragmática (Tópico, Foco y Contraste) al elemento copia: a) tópico: "es atribuido a un subacto con la función especial de señalar cómo el contenido comunicado se relaciona al registro construido gradualmente en el componente contextual" (Hengeveld y Mackenzie, 2008, p. 92); b) foco: es una estrategia del hablante, referente a la selección de la información nueva, relacionada con el contenido discursivo, lo que puede ocurrir tanto para (i) "rellenar un hueco informacional del oyente", como para (ii) "corregir la información equivocada que el oyente posee" (Hengeveld y Mackenzie, 2008, p. 89); c) contraste: es una función estructural informacional que "señala el deseo del hablante de enfatizar las diferencias particulares entre dos o más contenidos comunicados o entre un contenido comunicado e información disponible contextualmente" (Hengeveld y Mackenzie, 2008, p. 96).

3. Definitud del núcleo nominal: a) definido; o b) indefinido.

4. Naturaleza del elemento copia: a) pronombre tónico; b) pronombre átono; u c) otro.

5. Función sintáctica del elemento copia: a) sujeto, b) objeto directo; c) objeto indirecto; d) oblicuo; e) genitivo. 
En la próxima sección se presentan los resultados del análisis de los datos y su interpretación.

\section{ANÁLISIS Y DISCUSIÓN DE LOS DATOS}

Hay en los datos 3.466 casos de la cláusula relativa, siendo $96 \%$ de relativas restrictivas (3.232/3.466). De ese total, dispone del reasuntivo sólo 3\% (101/3.466) de los casos que serán analizados a continuación, conforme la aplicación de los criterios de análisis. Las relativas con el pronombre reasuntivo son casi exclusivamente introducidas por el pronombre relativo que, con excepción de dos casos con el que y dos con donde.

Los resultados encontrados confirman, en términos estadísticos, que la relativa no restrictiva es un contexto propicio al uso del reasuntivo. Se verifica que solo el 3\% (53/3.232) de los casos de relativas restrictivas en el corpus presenta pronombre de retoma. Las relativas no restrictivas disponen de reasuntivo en 35\% (48/133) de los casos. Esa distribución comprueba que hay una tendencia a que el reasuntivo sea una marca formal de la formulación de la relativa no restrictiva como un Acto. Eso acaba por activar un reflejo en el proceso de codificación de la relativa en español, no solamente en términos prosódicos, sino también en términos morfosintácticos. Esos resultados muestran que la pragmática ejerce influencia decisiva en el uso de la copia anafórica.

Con relación al tipo de reasuntivo, hay en el corpus predominancia de los pronombres átonos (lo, los, la, las, le, les) con índice de 79\%, según la Tabla I.

Tabla I. Distribución del tipo de relativa por tipo de reasuntivo en las relativas con copia anafórica.

\begin{tabular}{lrrrrrr}
\hline \multirow{2}{*}{ Tipo de reasuntivo } & \multicolumn{2}{c}{ Restrictiva } & \multicolumn{2}{c}{ No restrictiva } & \multicolumn{2}{c}{ Total } \\
\cline { 2 - 7 } & Frec. & $(\%)$ & Frec. & $(\%)$ & Frec. & \multicolumn{1}{c}{$\%$} \\
\hline Pronombre tónico & 2 & $67 \%$ & 1 & $33 \%$ & 3 & $3 \%$ \\
\hline Pronombre átono & 45 & $56 \%$ & 35 & $44 \%$ & 80 & $79 \%$ \\
\hline Otros & 6 & $33 \%$ & 12 & $67 \%$ & 18 & $18 \%$ \\
\hline Total & 53 & $52 \%$ & 48 & $48 \%$ & 101 & $100 \%$ \\
\hline
\end{tabular}

La alta frecuencia de los pronombres $l o(s), l a(s)$ y $l e(s)$ refleja lo que ocurre en la oración independiente, en la que esos pronombres están siendo usados como marcadores de concordancia de objeto (Franco, 1993), propuesta a la que también se afilia este estudio. Una ilustración de ese uso de reasuntivo como marcador de 
concordancia en la cláusula relativa se puede observar en (15) y (16).

En (15), el pronombre lo se refiere al antecedente algo, con el cual establece concordancia en género y número (masculino singular), señalando también la función sintáctica de objeto directo del verbo contar. En (16), una relativa de objeto indirecto, el pronombre le, que hace concordancia en singular con el antecedente, también marca la función sintáctica que este asume en la cláusula relativa. Se supone aquí que el uso del reasuntivo refleja cambios del sistema gramatical del español, que viene optando por expresar pronominalmente el complemento verbal. Los cambios en el sistema pronominal ocurren primeramente en la oración independiente, contexto más propicio para eso, y se expanden para la oración subordinada. Esa hipótesis precisa ser comprobada diacrónica y estadísticamente, así como fue hecho por Tarallo para el portugués brasileño (1983).

Del mismo modo que la redundancia de le(s) ocurre de modo categórico en las relativas, los pronombres $l o(s)$ y $l a(s)$ también se están naturalizando en las relativas de objeto directo, como una expansión de la recuperación a otras posiciones sintácticas. Es eso que se verifica al observar los resultados en lo concerniente a la función sintáctica relativizada, expuestos en la Tabla II.

Tabla II. Distribución del tipo de relativa por función sintáctica en las relativas con copia anafórica.

\begin{tabular}{lrrrrrr}
\hline \multirow{2}{*}{ Función sintáctica } & \multicolumn{2}{c}{ Restrictiva } & \multicolumn{2}{c}{ No restrictiva } & \multicolumn{2}{c}{ Total } \\
\cline { 2 - 7 } & Frec. & $(\%)$ & Frec. & \multicolumn{1}{c}{$(\%)$} & Frec. & $(\%)$ \\
\hline Sujeto & 4 & $50 \%$ & 4 & $50 \%$ & 8 & $8 \%$ \\
\hline objeto directo & 29 & $49 \%$ & 30 & $51 \%$ & 59 & $58 \%$ \\
\hline objeto indirecto & 19 & $73 \%$ & 7 & $27 \%$ & 26 & $26 \%$ \\
\hline Oblicuo & 1 & $14 \%$ & 6 & $86 \%$ & 7 & $7 \%$ \\
\hline Genitivo & - & - & 1 & $100 \%$ & 1 & $1 \%$ \\
\hline Total & 53 & $52 \%$ & 48 & $48 \%$ & 101 & $100 \%$ \\
\hline
\end{tabular}

El reasuntivo ocurre, en general, en las relativas de objeto directo e indirecto, pero, de modo similar a otros estudios, los datos aquí también apuntan para el uso del reasuntivo con las relativas de sujeto, que es la posición de más fácil relativiza- 
ción y, por lo tanto, la menos propicia a recibir el reasuntivo, según la jerarquía de accesibilidad de Keenan y Comrie (1977). Véanse los ejemplos a continuación, en los que hay la relativización de la posición sintáctica de sujeto, con la retoma del antecedente por medio del reasuntivo, sea en su posición canónica al principio de la oración, como en (17), o en el final de la oración, como en (18).

17 pues por cuestiones de trabajo de / de / de mi esposa / que / que $<$ alargamiento/ $>$ ella es costurera / pos ella es la / veces ella no tiene tiempo pa $<[\mathrm{r}]\rangle\langle[\mathrm{a}]\rangle / \mathrm{pa}<[\mathrm{r}]\rangle\langle[\mathrm{a}]\rangle$ atender a / a mis hijos (MONR_H21_041:1.236)

18 por supuesto gente que trabajan los dos / la pa <palabra_cortadal> la pareja / hh (MADR_H22_026:1.177)

Los datos discutidos hasta ahora suscitan dos cuestiones: ¿¿Para qué son usados los reasuntivos que no son pronombres átonos? ¿Cuál es la función del reasuntivo en las relativas de sujeto? Para intentar responderlas, véase la Tabla III, en la que los dos factores son contrastados.

Tabla III. Distribución del tipo de copia anafórica por función sintáctica en las relativas con reasuntivo.

\begin{tabular}{lcrrrrrrr}
\hline $\begin{array}{l}\text { Tipo de reasuntivo/ } \\
\text { Función sintáctica }\end{array}$ & \multicolumn{2}{c}{$\begin{array}{l}\text { Pronombre } \\
\text { tónico }\end{array}$} & \multicolumn{2}{c}{$\begin{array}{c}\text { Pronombre } \\
\text { átono }\end{array}$} & \multicolumn{2}{l}{ Otros } & \multicolumn{2}{c}{ Total } \\
\cline { 2 - 10 } & Frec. & $(\%)$ & Frec. & $(\%)$ & Frec. & $(\%)$ & Frec. & $(\%)$ \\
\hline sujeto & 2 & $25 \%$ & - & - & 6 & $75 \%$ & 8 & $8 \%$ \\
\hline objeto directo & - & - & 57 & $97 \%$ & 2 & $3 \%$ & 59 & $58 \%$ \\
\hline objeto indirecto & 1 & $4 \%$ & 22 & $85 \%$ & 3 & $12 \%$ & 26 & $26 \%$ \\
\hline oblicuo & - & - & - & - & 7 & $100 \%$ & 7 & $7 \%$ \\
\hline genitivo & - & - & 1 & $100 \%$ & - & - & 1 & $1 \%$ \\
\hline Total & 3 & $3 \%$ & 80 & $79 \%$ & 18 & $18 \%$ & 101 & $100 \%$ \\
\hline
\end{tabular}

Los pronombres oblicuos ocurren frecuentemente en la relativización de las posiciones sintácticas de objeto directo y de objeto indirecto. Otros tipos de elementos anafóricos aparecen en la relativización de la posición sintáctica de sujeto $(75 \%)$ y de oblicuo (100\%), preponderantemente. Esos elementos anafóricos pueden ser adverbios, sintagmas nominales, pronombres demostrativos o indefinidos, tal como los ejemplificados, respectivamente, en (19)-(22). 
21 una bronca tumultuaria que esa creo que fue la bronca más grande que se dio en la cuadra (LHAB_H12_037:1.178-179)

22 hay algunos quesalen <alargamiento/ $>$ to<alargamiento/ $>$ dos toma $<$ alargamiento/ $>$ dos (MONV_H11_035:1.917)

La idea defendida aquí, suscitada por el estudio de Cerrón-Palomino, es la de que esos reasuntivos son usados como soporte para la atribución de función pragmática (2006), como se comprueba en la Tabla IV.

Tabla IV. Distribución del tipo de pronombre copia por atribución de función pragmática en las relativas con copia anafórica.

\begin{tabular}{lcrrrrrrr}
\hline $\begin{array}{l}\text { Reasuntivo/función } \\
\text { pragmática }\end{array}$ & \multicolumn{2}{c}{$\begin{array}{c}\text { Pronombre } \\
\text { tónico }\end{array}$} & \multicolumn{2}{c}{$\begin{array}{c}\text { Pronombre } \\
\text { átono }\end{array}$} & \multicolumn{2}{c}{ Otros } & \multicolumn{2}{c}{ Total } \\
\cline { 2 - 9 } & Frec. & $(\%)$ & Frec. & $(\%)$ & Frec. & $(\%)$ & Frec. & $(\%)$ \\
\hline Tópico/Contraste & 2 & $25 \%$ & - & - & 6 & $75 \%$ & 8 & $8 \%$ \\
Foco & - & - & - & - & 8 & $100 \%$ & 8 & $8 \%$ \\
Sin función prag. & 1 & $1 \%$ & 80 & $94 \%$ & 4 & $5 \%$ & 85 & $84 \%$ \\
Total & 3 & $3 \%$ & 80 & $79 \%$ & 18 & $18 \%$ & 101 & $100 \%$ \\
\hline
\end{tabular}

Los resultados muestran que el pronombre prototípico de las relativas con reasuntivo a las que no se atribuye función pragmática es codificado como forma átona. Por otro lado, la necesidad de atribuir la función pragmática al referente del antecedente en la relativa activa el uso de adverbios, sintagmas nominales, pronombres demostrativos o indefinidos como soporte para la función pragmática. Se consideren los ejemplos en (23) y (24).

23 sí / eran / cinco materias que se llaman de tronco común / que son<alargamiento/> $<$ vacilación/> / que esas se pueden exonerar<alargamiento/> / exoneré cuatro / con nueve / porque es con nueve que se exonera <simultáneo $>\mathrm{y}</$ simultáneo $>$ (MONV_H13_021:1.107-108)

En esos casos, los reasuntivos esas y ahí reciben función pragmática tópico, pues se refieren al modo como el contenido comunicado se relaciona con el registro construido gradualmente en el componente contextual (Hengeveld y Mac- 
kenzie, 2008, p. 92); en otros términos, los reasuntivos se refieren a los referentes materias y paila, que, una vez introducidos previamente en la oración anterior, están disponibles contextualmente en la memoria de corto plazo y están disponibles para los interlocutores. A partir de la información recuperada como tópico, el hablante construye su enunciado yendo de la información dada a la información nueva. Martínez-Caro (1997) analiza construcciones independientes semejantes a esas como tópicos enfáticos. In.: Eso actualmente no lo puedo, no lo puedo señalar (Martínez-Caro, 1997, p. 1)

Para la autora, el pronombre eso, que retoma un tópico introducido antes en el discurso, está posicionado en el inicio de la oración para señalar su carácter enfático. Esa posición, denominada $\mathrm{P}^{\mathrm{I}}$, es destinada a los constituyentes con función pragmática. Eso ocurre en los casos en los que el argumento del verbo no está ubicado en su posición "natural", usualmente después del verbo. En caso de que no hubiera la necesidad de enfatizarlo, el pronombre no estaría expreso.

En los casos de reasuntivo con la relativa de sujeto, se defiende que el pronombre también recibe la función pragmática de tópico/contraste. Esa interpretación armoniza con la de Fanjul (2014), según la cual la posibilidad de que un pronombre sujeto en español ocurra acarrea un efecto de contraste como resultado de un proceso relacionado con la progresión de la información y a la identificación de la referencia. En el ejemplo (17), retomado como (26) por conveniencia, se percibe que el reasuntivo ella retoma el antecedente esposa, en contraste con otro referente presente en el contexto $(y o)$.

26 A: ¿y<alargamiento/> usted qué tan bueno es / para cocinar? / ¿no / no cocina / o no / o sea?

B: no<alargamiento/> sí / sí cocino o sea<alargamiento/> / de repente // este $<$ alargamiento/ $>/ /$ pues por cuestiones de trabajo de / de / de mi esposa / que / que <alargamiento/ $>$ ella es costurera / pos ella es la / veces ella no tiene tiempo $\mathrm{pa}<[\mathrm{r}]><[\mathrm{a}]>/ \mathrm{pa}<[\mathrm{r}]><[\mathrm{a}]>$ atender a / a mis hijos $($ MONR_H21_041:1.236)

Todos los reasuntivos con función pragmática tópico se encuentran en $\mathrm{P}^{\mathrm{I}} \mathrm{y}$ pueden o no recibir también la función pragmática contraste. En el caso (23), citado anteriormente, esas, que es tópico, establece un contrapunto con otras materias que no se pueden eliminar, recibiendo también la función pragmática contraste; en (24), a su vez, ahí tiene sólo función tópica de reactivación, pero sin establecer el contraste entre referentes.

Los reasuntivos también pueden recibir la función foco, que es usada como una estrategia para seleccionar la información nueva con relación al contenido 
discursivo, como en el ejemplo (20), repetido aquí como (27) por conveniencia.

me imagino que son<alargamiento/ $>$ parejas que $<$ alargamiento/ $>$ de mediana edad no suelen ser gente joven / que ha vendido su primera vivienda aprovechando también la subida y aprovechan para dar la entrada ¿no? / hh por su <palabra_cortada/> por supuesto gente que trabajan los dos / la pa <palabra_cortadal> la pareja / hh B: hm / sí y ¿ha ido ha venido mucha gente nueva al barrio? //

A: $s<$ alargamiento/>í / sí ha venido gente nueva pero // eeh no <vacilación/> no $<$ vacilación/> no te podría decir cómo es esta gente nueva que ha venido ¿no? / también porque<alargamiento/> en los últimos años yo he estado más // sumergido en mi trabajo y<alargamiento/> no he compartido mucho la vida de barrio ¿no? pero // hh / hm<alargamiento/ $>\mathrm{mm}$ s<alargamiento/ $>$ í / ha venido gente<alargamiento/ $>$ / nueva / yo / pienso // <vacilación/ $>$ sí que han sin que tenga mucho valor este $\mathrm{p}<$ palabra_cortada/ $><$ vacilación/ $>$ esta opinión ¿̨no? pero que es gente no muy joven / estamos hablando a lo mejor de cuarenta cuarenta y pico años ¿no? // y<alargamiento/> //una pareja que trabajan los dos (MADR_ H22_026:1.185)

En ese ejemplo, el reasuntivo los dos es el sujeto de la oración, pero está ubicado en la posición final, señalando su carácter focal. Los interlocutores están hablando sobre cómo la gente nueva que fue a vivir en el barrio de A puede pagar por una habitación muy cara. En ese contexto, el hablante focaliza la información que es más importante en la oración, la de que los dos trabajan para poder pagar la casa. En español, el cambio del constituyente de su posición "natural” para la posición final es una estrategia de marcación de foco (Martínez-Caro, 2011, pp. 3-4).

De esa forma, se percibe que el papel de la pragmática es decisivo para el uso del reasuntivo, sea debido a la formulación pragmática de la no restrictiva o a la necesidad de atribución de función pragmática al referente dentro de la cláusula relativa. Esas motivaciones abarcan la mayoría de los casos en el corpus; sin embargo, es preciso explicar también el uso del reasuntivo en las relativas restrictivas, contexto que generó dudas inicialmente en cuanto a la clasificación de la relativa. Inicialmente, la idea era la de que, si hay un núcleo que precisa ser definido, todos los sintagmas y oraciones que se refieren a él contribuyen para la construcción del referente y, por lo tanto, funcionan como modificadores simples o complejos (relativa restrictiva). Sin embargo, al volver a los datos, para intentar entender por qué el reasuntivo ocurría con la relativa restrictiva, se percibe que normalmente el núcleo (indefinido) estaría ya definido por lo menos por un modificador, antes de ser modificado por la relativa con reasuntivo. Eso ha impuesto el siguiente cuestionamiento: ¿Cuántas informaciones el hablante siente la necesidad de atribuir al núcleo, para especificarlo? Se verifica que gran parte de los casos de relativa en ese contexto no son, de hecho, casos de relativa restrictiva, por eso fueron analizadas 
aquí como relativas no restrictivas. Se trata, en efecto, de construcciones en las que el núcleo ya se encuentra definido por modificador simple o complejo, como las ejemplificadas en (28) y (29), respectivamente.

A: y los amigos que tenés aho<alargamiento/>ra ¿̨ónde los conociste? / los amigos B: uhm

A: que has hecho como adulto

B: algunos // en el liceo / eeh // que no los veo mucho / (MONV_H23_019:1.339)

29 I: también el / pues estaciones de pop / de rock / de trova / esta estación / una / hay una estación que me gusta / que no la traigo en el carro porque no sé / no / no no / sé bien / no pesca bien el cuadrante (MONR_H13_025:1.986)

En ese caso, se defiende que, si el núcleo indefinido ya fue previamente especificado por otro modificador, la cláusula relativa puede ser no restrictiva o, por lo menos, no tiene el mismo nexo que tendría una relativa restrictiva que es el único modificador del núcleo. Se decidió, por lo tanto, analizar esas construcciones como relativas no restrictivas.

En (28), el núcleo amigos ya fue referido anteriormente en el discurso por A, que lo especificó antes por la relativa restrictiva que tenés ahora y más adelante por la relativa restrictiva que has hecho como adulto, o sea, entre el conjunto de amigos de B, A sólo desea saber sobre aquellos que habían sido hechos después de el tornarse adulto, y no los que habían sido hechos en la infancia. En el cuarto turno, en que está elíptico, el núcleo amigos es modificado por un pronombre indefinido (algunos). Se cree que la relativa que no los veo mucho no tenga la función de especificar el núcleo, pero añadir una información adicional, que el hablante cree que es relevante en la situación de comunicación. Este tipo de construcción trae incluso la idea de ruptura de expectativa (son amigos, pero no los veo mucho).

De manera semejante, el núcleo una estación, en (29), ya se encuentra especificado por la cláusula relativa restrictiva que me gusta. Como la segunda relativa, que no la traigo en el carro, trae una información adicional, ella puede ser interpretada como no restrictiva. Hay también, en ese caso, una contrariedad de la expectativa (me gusta la estación, pero no la tengo en el carro).

Esta posición incluyó, también, la construcción presentativa discutida por Suárez-Fernández (2013), que se ejemplifica en (14) y se repite aquí como (30) por conveniencia.

Mi hermana tenía una cartera blanca que siempre peleábamos por ella.

En (30), el núcleo una cartera ya se encuentra definido por el modificador blanca. Para la autora, el papel de la relativa que siempre peleábamos por ella es traer 
una información adicional que el hablante juzga relevante introducir en el discurso para atingir sus objetivos. Esas construcciones son distintas y, por la definición de la autora, se puede pensar que son parecidas a la relativa no restrictiva, ya que es esa que tiene la función de traer información adicional sobre el núcleo. Si se acepta ese punto de vista, esas construcciones pueden ser analizadas como actos discursivos, lo que por sí ya es motivo para el uso del reasuntivo.

La muestra contiene una construcción presentativa semejante también a lo que Suárez-Fernández (2013, p. 88) interpreta como "comentario subjetivo" sobre el núcleo (Tienes una hija / que no te la mereces).

A: trabajabas de lunes aaa viernes

B: de lunes a viernes de <alargamiento/ $/$ pues al final / de siete la mañana a diez de la noche / sin comer / y<alargamiento/> los viernes en principio te vas a las tres // yo no me iba ninguno hasta que me dieron el sobre y dije $<$ cita $>$ pues ahora me voy a las tres tú $</$ cita $>/ /<$ entre_risas $>$ fue un acto de protesta $</$ entre_risas $><$ risas = "todos"/ $>/ /$ no pero una cosa es el horario oficial de las empresas $y<$ alargamiento/ $>$ / y otra cosa es lo que se cumple y<alargamiento/> en las empresas se hace lo que hacen los jefes si los jefes se van a las tres todo el mundo se va a las tres // jjoder! pues que empiecen a cumplirlo ellos / [... $]^{5}$ pero es que ya al final no sabía ni lo que hacía o sea / tú empiezas a trabajar a las siete de la mañana y a las <vacilación/> lo que $\mathrm{t}<$ palabra_cortada/ > <vacilación/> te estás leyendo un pliego / de la Comunidad autónoma de Cataluña en catalán por supuesto / a las diez de la noche es que no sabes lo que estás haciendo // yo no sé <risas = "I"/> // por qué / luego acertaba / porque / hay cosas que no lo comprendo cómo / si es que no me estaba enterando de lo que estaba leyendo (MADR_H13_013:1.812)

Se percibe que, en (31), la relativa no tiene función especificadora o identificadora. Se trata, en efecto, de un comentario subjetivo sobre el núcleo cosas, que resume todo el contenido mencionado anteriormente, en que $\mathrm{B}$ narra las pésimas condiciones de trabajo (trabajaba varias horas, sin almuerzo). El núcleo cosas, a pesar de ser genérico, deja implícita su modificación (cosa difícil, aburrida, penosa etc.), como la paráfrasis hay cosas horribles que no comprendo cómo (puede haber). También se defiende que las construcciones de ese tipo pueden ser analizadas como relativas no restrictivas. La interpretación dada por Suárez-Fernández corrobora este análisis:

Por lo que se refiere a las de nominal indefinido, se observa que se aproximan a las explicativas en varios aspectos, entre ellos, en la evidencia de que no participan en la identificación del referente, puesto que es información nueva, por tanto, presen-

\footnotetext{
${ }^{5}$ Aquí se suprimió parte del texto.
} 
tada por el hablante como no accesible, y en que, al menos en ocasiones, poseen particularidades entonacionales que manifiestan menor trabazón entre antecedente y relativa, por lo que aparece con facilidad el reasuntivo. (Suárez-Fernández, 2013, p. 89)

La diferente función desarrollada por esas construcciones y la presencia de aspectos prosódicos delineantes contribuyen para comprobar que se trata de relativas no restrictivas, que son formuladas pragmáticamente como actos, codificadas con pronombre reasuntivo en el nivel morfosintáctico y con variación prosódica en el nivel fonológico.

Es probable que algunos de los casos de reasuntivo con la relativa restrictiva, atestados en la Literatura, puedan haber sido interpretados equivocadamente en los diversos estudios sobre el tema, dirección que también este estudio había tomado al comienzo. Se cree que los argumentos dados son suficientes para comprobarse que esas construcciones son de facto relativas no restrictivas y así se hace el análisis aquí.

Por fin, queda por explicar los 3\% del uso de reasuntivo en las relativas restrictivas encontrados en el corpus. Esos casos son explicados mayoritariamente por razones de procesamiento de la información, debido a algunos factores que generan dificultad en la identificación del referente. El uso del reasuntivo en las relativas restrictivas se debe a la inserción de otros sintagmas u oraciones entre el núcleo y la posición que ocupa en la relativa, como en (32) y (33), respectivamente.

yo siempre cito<alargamiento/> a un profesor de la universidad que / cuando ocurrió el problema de pavimentación en la Alameda / eeh él salió lo entrevistaron (SCHI_H13_073:1.604-605)

La inserción de constituyentes entre el núcleo y su posición en la cláusula relativa provoca el alejamiento entre el reasuntivo y su referente, lo que hace necesario el uso del reasuntivo. En (32) y (33), la inserción de todos los años y cuando ocurrió el problema de pavimentación en la Alameda puede contribuir para la dificultad cognitiva de reactivación del referente, lo que activa la inserción del reasuntivo como un mecanismo de facilitación del procesamiento. El factor distancia se discutió en diversos estudios (Silva-Corvalán, 1996; Cerrón-Palomino, 2006; Suaréz-Fernández, 2013), que lo consideran crucial en la explicación del uso del reasuntivo.

El uso del reasuntivo también puede ser motivado por el propio proceso de elaboración de la cláusula, lo que, hasta donde se sabe, aún no fue explorado en las investigaciones sobre el tema. Se percibe, debido a la ocurrencia de repetición 
vacilante y/o alargamiento, que el hablante aún no está seguro de lo que va a decir, elaborando y produciendo su discurso al mismo tiempo, como se ejemplifica en el caso (34), en el que hay la repetición del pronombre relativo, junto al alargamiento del segundo que.

34 pero ya un amigo que que<alargamiento/> / que lo tenís ya adulto / ya es diferente (SCHI_H12_037:1.406)

De los 53 casos de las restrictivas con reasuntivo, 16 son ocurrencias del pronombre oblicuo le, que ocurre categóricamente para la posición sintáctica de objeto indirecto.

Solo cinco casos no son explicados por el uso categórico de le y por los factores de procesamiento. No se cree que eso sea un problema, por el contrario, apenas atestigua el carácter mutable y no discreto de la lengua. Se presentan esos casos a continuación.

35 es algo que lo tenemos al alcance de la mano <simultáneo> ¿no? (MADR_ H22_026:1.115-116)

tengo dos amigos que los conocí de niño / Alain y Yosmán (LHAB_ H11_001:1.302-303)

38 veo que la gente que la educaron en el campo es una gente muy solidaria (MEDE_ H23_001:1.235)

39 había una barra que la colocamos la barra del <énfasis>Che Guevara</énfasis> I (MEDE_H23_001:1.549)

Se intenta, en esa sección, explicar el uso del reasuntivo, considerando las motivaciones pragmáticas que lo influyen, para entender por qué esa estrategia tiende a ser usada en contextos específicos (relativa no restrictiva, relativización de la posición sintáctica de objeto directo, restrictivas modificando núcleos indefinidos). A continuación se sintetizan las principales conclusiones.

\section{CONCLUSIONES}

En este estudio se defiende que hay una tendencia de que el reasuntivo sea una marca morfosintáctica de la diferencia en la formulación de las relativas restricti- 
va y no restrictiva. A diferencia de la restrictiva, que funciona como un modificador del núcleo en el nivel representacional, la relativa no restrictiva es formulada como un acto discursivo con la función retórica de adición de información sobre el referente. Esa diferencia se refleja no sólo en la codificación fonológica de las relativas, como lo sostenido por Hengeveld y Mackenzie (2008), sino también en la codificación morfosintáctica, en la que se puede insertar un reasuntivo. El Esquema-resumen 1 muestra los principales postulados desarrollados a lo largo de este texto.

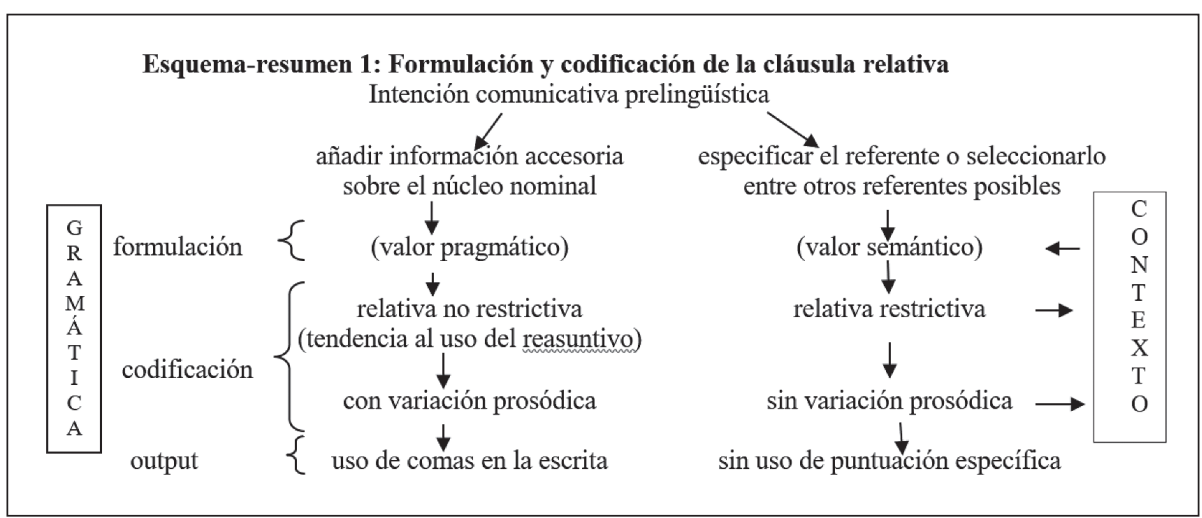

Esquema 1. Formulación y codificación de la cláusula relativa.

Es baja la frecuencia de uso del reasuntivo en la relativa restrictiva y los pocos casos reflejan la dificultad del procesamiento de la información, la necesidad de la especificación del núcleo nominal indefinido y el uso categórico del pronombre átono le, que es usado "redundantemente de manera natural" tanto en la restrictiva como en la no restrictiva (Trujillo, 1990, p. 38), reflejando lo que viene ocurriendo en la oración independiente.

Este estudio también ofrece una interpretación a la alta frecuencia de reasuntivo en la relativa restrictiva con antecedente indefinido, proponiendo que esas construcciones, en algunos contextos, son actos discursivos formulados pragmáticamente, lo que llevó a interpretarlos como relativas no restrictivas.

Se observa también la existencia de un diferente funcionamiento de los pronombres oblicuos $l o(s)$, la $(s)$ y $l e(s)$, en comparación con otros tipos de reasuntivo. Los pronombres átonos se están especializando como marcas morfosintácticas de la distinta formulación de las relativas restrictivas y no restrictivas. Adverbios, pronombres demostrativos, pronombres indefinidos y sintagmas, por su lado, son usados como soporte para la atribución de función pragmática al reasuntivo en la relativización de posiciones sintácticas específicas. Cuando se atribuye la función 
pragmática tópico, el reasuntivo se coloca en la posición inicial y también puede asumir la función pragmática de contraste. Ya cuando se atribuye la función pragmática de foco al reasuntivo, este aparece en la posición posverbal. Se presentan estos resultados en el Esquema-resumen 2.

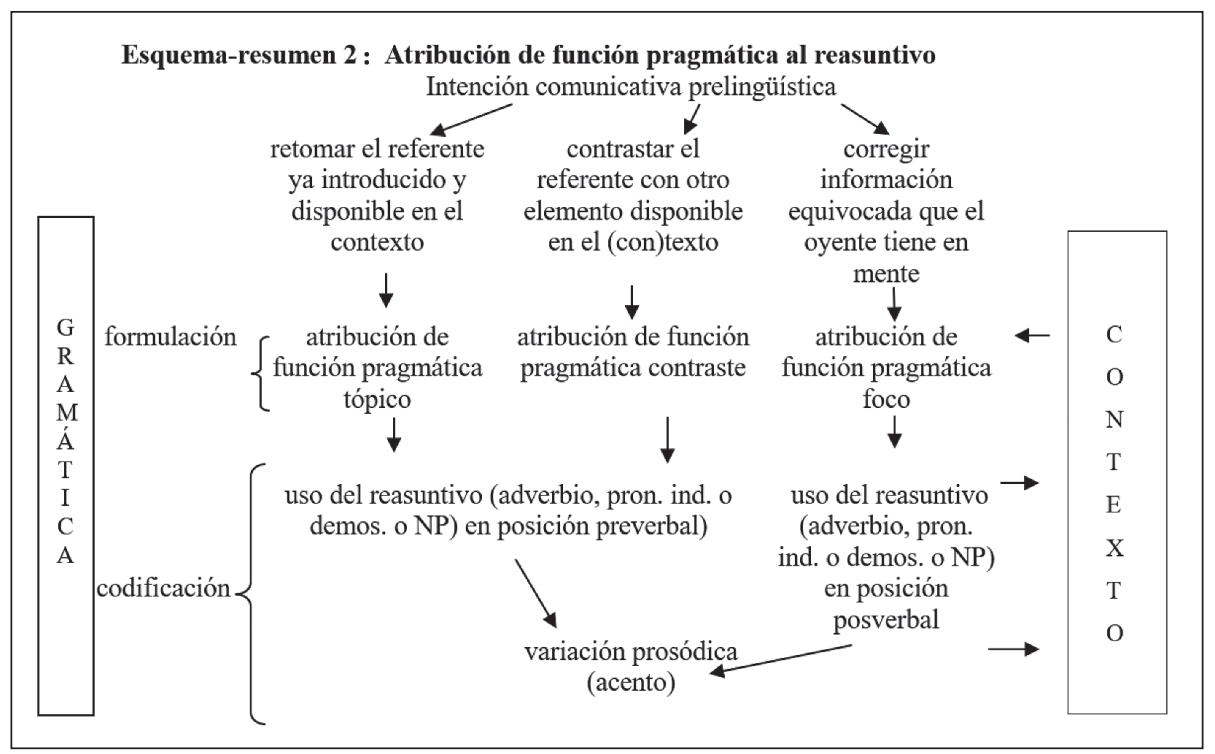

Esquema 2. Atribución de función pragmática al reasuntivo.

Al fin y al cabo, la propuesta aquí defendida tiene como propósito fomentar los estudios desarrollados sobre la cláusula relativa en español, tema complejo que tiene motivadas las diversas explicaciones para el uso del reasuntivo. El punto de vista a partir del cual se observa el fenómeno determina, en gran medida, la interpretación de los datos. Motivados por un modelo gramatical en el que el componente gramatical engloba los aspectos pragmáticos, semánticos, morfosintácticos y fonológicos, se cree que este estudio contribuyó a la comprensión del uso del reasuntivo en la cláusula relativa, yendo más allá de un análisis exclusivamente semántico y señalando el papel que cada nivel desarrolla en el uso de ese fenómeno lingüístico. Por supuesto, el uso del reasuntivo dispone de algunas motivaciones que aún necesitan ser plenamente investigadas, como la construcción del referente en el discurso y la distancia entre el núcleo y su posición en la relativa. Además, motivados por Tarallo (1983), se cree que un estudio diacrónico que relacione la pronominalización y la relativización en español puede esclarecer cómo los cambios ocurridos en el sistema pronominal en la oración independiente influyen en los cambios del sistema de relativización. 


\section{REFERENCIAS}

Bentivoglio, P. (2003). Las construcciones 'de retoma' en las cláusulas relativas: un análisis variacionista. En F. M. Fernández, J. A. S. Padilla, M. Vaquero, M. L. G. Arais, C. H. A. y F. Gimeno-Menéndez (Eds.). Lengua, Variación y Contexto, Estudios dedicados a Humberto López Morales (pp. 507-520, Tomo II). Madrid: Arco Libros.

Borzi, C., y Morano, M. (2009). Cláusulas relativas con duplicación del objeto. Onomazéin, 19, 71-88.

Brucart, J. M. (1999). La estructura del sintagma nominal: las oraciones de relativo. En I. Bosque, y V. Demonte (Coords.), Gramática Descriptiva de la Lengua Española (pp. 395-522, Tomo I). Madrid: Espasa.

Caviglia, S. y Malcuori, M. (2007). Las oraciones de relativo en la interfaz gramática-discurso. En V. Bertolotti et al. (eds.) Estudios de Lingüistica Hispánica (pp. 43-69). Cádiz: Universidad de Cádiz.

Cerrón-Palomino, Á. (2006). Pronombres de retoma en cláusulas relativas del castellano peruano: un fenómeno de causación múltiple. Lexis, 29, 231-258.

Cerrón-Palomino, Á. (2015). Resumption or contrast? Non-standard subject pronouns in Spanish Relative Clauses. Spanish in Context, 12, 349-372.

Dassinger, L., y Toupin, C. (1994). The development of relative clause functions in narrative. En R. A. Berman, y D. I. Slobin (Eds.), Relating events in narrative. A crosslinguistic Development Study (pp. 457-514). Hillsdadle, New Jersey: Lawrence Erlbaum.

De Mello, G. (1992). Duplicación del pronombre relativo de objeto directo en el español hablado culto de once ciudades. Lexis, XVI-1, Lima, Pontifica Universidad Católica del Perú.

Fanjul, A. P. (2014). Conhecendo assimetrias: a ocorrência de pronomes pessoais. En A. P. Fanjul, y N. M. González. Espanhol e português brasileiro: estudos comparados (pp. 29-50). São Paulo, Brasil: Parábola Editorial.

Franco, J. (1993). On object agreement in Spanish. USC Tesis doctoral. Los Angeles: GSIL Publication Series.

Gibson, E. (1998). Linguistic complexity: locality of syntactic dependencies. Cognition, 68, 1-76.

Hengeveld, K., y Mackenzie, J. L. (2008). Functional discourse grammar: a typologically-based theory of language structure. Oxford: Oxford University Press.

Keenan, E. L.; y Comrie, B. (1977). Noun phrase accessibility and universal grammar. Linguistic Inquiry, Cambridge, 8, 63-99. Disponible en http://lingo. stanford.edu/sag/L222B/papers/KeenanComrie.pdf. [Consulta: 18/04/2017].

Martínez-Caro, E. (1997). Tópicos retomados enfáticamente en español y en inglés. XV Congreso de la Asociación Española de Lingüistica Aplicada (AESLA), Universidad de Zaragoza, España. Disponible en https://www. 
academia.edu/1990500/Funciones_pragm\%C3\%A1ticas_y_estructura_sint $\%$ C3\%A1ctica_en_espa $\% \mathrm{C} 3 \% \mathrm{~B} 1 \mathrm{ol}$.t $\% \mathrm{C} 3 \% \mathrm{~B} 3$ picos_retomados_ enf\%C3\%A1ticamente_1998_. [Consulta: 18/04/2017].

Martínez-Caro, E. (2011). Posición final y orden de constituyentes en español. Conferencia sobre los estudios descriptivos de la lengua española. Universidade Estadual Paulista: São José do Rio Preto.

PRESEEA. (2014). Corpus del Proyecto para el estudio sociolingüistico del español de España y de América. Alcalá de Henares, Universidad de Alcalá. Disponible en http://preseea.linguas.net. [Consulta: 18/04/2017].

Silva-Corvalán, C. (1996). Resumptive pronouns: a discourse explanation. En C. Parodi, C. Quicoli, M. Saltarelli, y M. L. Zibizarreta. Aspects of Romance Linguistics (pp. 383-395). Washington D. C.: Georgetown University Press.

Suárez-Fernández, M. (2010). Cláusulas de relativo con pronombre personal anafórico en castellano medieval. Nueva Revista de filología hispánica, 58, 1-37.

Suárez-Fernández, M. (2013). Funcionalidad del pronombre reasuntivo en cláusulas relativas en español. Moenia, 19, 63-96.

Tarallo, F. L. (1983). Relativization strategies in brazilian Portuguese. PhD Dissertation. Philadelphia: University of Pennsylvania.

Trujillo, R. (1990). Sobre la supuesta despronominalización del relativo. Estudios de Lingüistica, Universidad de Alicante, 6, 23-46.

Zabalegui, N. (2005). Análisis del pronombre de retoma en las cláusulas relativas en documentos de Venezuela escritos en los siglos XVI al XVIII. Actas del XIV Congreso Internacional de la Alfa, Monterrey, México. Disponible en http:// www.mundoalfal.org/cdcongreso/cd/indice_linguistica_historica.html. [Consulta: 18-04-2017]. 